\title{
Formation postgraduée: le point de la situation dans le canton de Vaud en 2011
}

\begin{abstract}
Au 1.1.2011 est entré en vigueur le nouveau programme de formation postgraduée en médecine interne générale qui remplace le programme de médecine générale et le programme de médecine interne. Sur cinq ans, ce programme permet de se former tant en médecine de ville qu'en médecine de campagne ou en médecine hospitalière (réf: BMS/2010;91:49/pp 1929-1932). Durant cinq ans des dispositions transitoires sont prévues. La souplesse et la liberté offerte par ce programme permettent à chacun de trouver sa voie en fonction de son projet professionnel mais nécessite souvent un accompagnement pour sa conceptualisation et sa cohérence. C'est là que les médecins-chefs, les maîtres de stage et le Cursus Romand de Médecine Générale (CRMG) jouent leur rôle.

Le CRMG participe à la promotion de cette formation auprès des étudiants et des assistants. Nous avons actuellement des contacts réguliers avec une centaine de jeunes collègues. Le succès va grandissant, de pair avec la visibilité de l'IUMG et l'introduction du stage au cabinet en $6_{\text {ème }}$ année. Nous travaillons dans la PMU qui nous facilite le travail. Le lien direct du CRMG avec le programme vaudois d'assistanat au cabinet médical est fondamental. Ce dernier est piloté par le Dr Patrick Cuénoud de Lausanne. Presque tous les postes sont occupés et les assistants intègrent bien la nécessité de cet apprentissage. Le besoin en maîtres de stage va augmenter d'autant que le nouveau programme de formation insiste sur cet assistanat. Avis aux amateurs!

Par ailleurs nous participons au développement de formations périphériques intégrées. Dans une région, autour d'un hôpital, des autres structures sanitaires et des cabinets, les assistants travaillent deux à trois ans avec l'aide et la coordination d'un praticien ins-
\end{abstract}

Coordinateurs du Cursus Romand de Médecine Générale (CRMG). tallé. Le pari est de favoriser l'installation des collègues en périphérie. Le programme dans le Nord vaudois a démarré sur les chapeaux de roue avec plus de dix assistants engagés. Le poste de coordinateur pour la région de Nyon est actuellement au concours et le projet va démarrer cette année. II n'y a pas encore de projet sur la Riviera, dans le Chablais ou dans la Broye.

En résumé les différentes instances, dont l'AMOV, collaborent efficacement au profit de la formation postgraduée et les étudiants sont de plus en plus motivés à embrasser notre métier. Cela ne pourrait se faire sans le soutien de la Santé Publique. Le retard pris ne pourra être comblé mais nous croyons ainsi amortir la pénurie de médecins de famille. L'importance de l'encadrement et de l'engagement de la Faculté et de I'IUMG pour la formation postgraduée augmente de façon évidente. Cela demandera des forces et une ouverture toujours plus grande de nos cabinets comme lieu de formation.

Correspondance:

Dr Bernard Giorgis

Spécialiste en médecine générale $\mathrm{FMH}$

20, Chemin des Esserpys

1032 Romanel-s-Lausanne

bernard.giorgis@hospvd.ch 